\title{
The importance of the epigenome for social-related neural circuits
}

\author{
Ryan V Wheeler ${ }^{1}$ \& Tamara B Franklin*,1 \\ ${ }^{1}$ Department of Psychology \& Neuroscience, The Social Lab, Dalhousie University, Halifax, NS B3H 4R2, Canada \\ *Author for correspondence: tamara.franklin@dal.ca
}

First draft submitted: 5 September 2019; Accepted for publication: 6 September 2019; Published online: 8 November 2019

Keywords: DNA methylation $\bullet$ histone deacetylase $\bullet$ neurocircuitry $\bullet$ rodent $\bullet$ social behavior

Social mammals, including rodents and humans, depend on active participation in numerous social interactions on a daily basis. These include maternal behaviors, sexual behavior, pair bonding and peer-to-peer interactions, all of which are critical to our survival and reproductive fitness. These complex behaviors require the integration of many neural systems (i.e., chemosensory, visual, auditory and somatosensory information) and a growing number of studies in both social insects and mammals have suggested that epigenetic mechanisms may play an important role in maintaining normal social functions [1]. However, whether dynamic changes in epigenetic regulation are recruited to mediate day-to-day social interactions in social mammals remain to be seen. Additionally, whether epigenetic regulation is critical for the plasticity required for social circuits to adapt to the array of social situations presented is still unknown. We focus here on the roles that DNA methylation and histone acetylation play on social behaviors, and what that might mean for social circuits in the brain.

\section{Brain circuits implicated in social function}

Social interactions between two conspecifics require arguably the most complex neural processing required of a social mammal. Engagement with a social stimulus often requires receiving and emitting cues in multiple sensory modalities to relate to a conspecific who themselves are receiving and emitting social cues. The range of possible behavioral outputs by both individuals creates a situation that is highly unpredictable compared with nonsocial processing. In addition, the appropriate behavioral output is also likely context specific. Despite its complexity, social decision making must be performed on a short timescale and the incoming cues are highly dynamic and can quickly change. Thus, it is not surprising that numerous social circuits involving many different brain areas have been identified in both rodents and humans. It is still unclear if neural circuits exist that are uniquely associated with social behaviors or the extent to which social circuits overlap with nonsocial circuits.

In general, many of the described social circuits in rodents begin with chemosensory input to the olfactory bulb, after which the information flows through amygdala nuclei to the bed nucleus of the stria terminalis to hypothalamic nuclei and ultimately to the periaqueductal gray and other midbrain regions that mediate the behavioral output [2]. While much of the research has focused on the olfactory bulb as the primary social sensory system in rodents, multiple sensory components are likely needed to be integrated for an appropriate behavioral response during a social interaction; for instance, ultrasonic vocalizations, visual input and chemosensory cues are all components of male-female interactions in rodents. Currently, areas of the amygdala have been suggested to be key sites for multisensory integration. Circuits involved in generating innate social behaviors have been well described in a recent review by Chen and Hong [2] and will not be discussed in detail here.

Other areas, like the hippocampus and the prefrontal cortex, have emerged as important brain regions for social behaviors that are modulated by past experiences. The same neuronal population in the dorsal CA2 subregion of the hippocampus projects to both the ventral CA1 subregion [3,4], a pathway important for social recognition memory, and to the lateral septum, a pathway which ultimately leads to the disinhibition of the ventromedial hypothalamus and aggression, a behavior influenced by past social experiences and social recognition [4]. The prefrontal cortex has also been shown to play an important role in social decision making as well as the plasticity needed for a learned social response [5]. The projection from the dorsal prefrontal cortex to the dorsal periaqueductal gray plays a critical 
role in social avoidance, and negative social experiences can weaken afferent inputs to the prefrontal cortex from the mediodorsal thalamus.

\section{Histone acetylation \& social function}

Histone acetylation modulates a range of social behaviors which are critical for survival and reproduction, including both the early programming of sexual behaviors as well as experience-dependent changes in female sexual behavior, maternal care and partner preference formation during adulthood. These findings are the results of experiments inhibiting histone deacetylase (HDAC) activity and observing subsequent behavior. Inhibition of global HDAC activity (using intracerebroventricular administration of trichostatin A) or specific HDAC2 or HDAC4 downregulation (using antisense oligodeoxynucleotides) in rat pups during the critical period of sexual differentiation that occurs in postnatal development results in abnormal masculinization of the brain and subsequent male sexual dysfunction [6]. In females, oral administration of an HDAC inhibitor, sodium butyrate, increases experiencedependent lordosis receptivity in sexually naive mice [7]. HDAC activity is also important for experience-dependent changes in maternal behavior. Here, treatment with sodium butyrate in sexually naive female mice, exposed to pups for two consecutive days, promotes the maternal care performed on a further pup exposure occurring 1 month after the initial treatment [8]. In prairie voles, a rodent which displays a monogamous mating system, intracerebroventricular administration of trichostatin A encourages the formation of partner preference [9]. In all of these examples, the global changes in histone acetylation was associated with specific changes in expression of genes known to be important in social function (i.e., estrogen, oxytocin or vasopressin receptors) within brain areas previously linked to social circuits (i.e., hypothalamic nuclei), but the causal link between region-specific changes in histone acetylation of these specific genes and behaviors is still lacking. Clearly, there is ample room to increase our understanding of the role of histone modifications in social function and, in particular, to begin examining how cell-type- and region-specific histone regulation affects the expression of social behavior repertoires. However, evidence thus far does support the concept that epigenetic regulation of the transcriptome by histone acetylation is not only important for normal neural development related to social behavior but also modulates learning and memory related to social experiences and future social decisions. This plasticity is likely dependent on altered circuit function but this has not been explicitly explored.

\section{DNA methylation \& social function}

Due to the traditional belief that DNA methylation is a highly stable epigenetic mechanism for gene regulation, research into the impact of differential DNA methylation on social behavior has predominantly focused on the persistent effects of an individual's early social environment. For instance, an early life social stress resulting from maternal separation impacts DNA methylation in brain and sperm cells [10-13] as well as future social behaviors [14]. While many of these studies are correlational, finding that early life social stress decreases hypothalamic methylation in a downstream enhancer region of a key regulator of social behavior (arginine vasopressin), and increases its gene expression, strongly suggest that an early social stress can result in early programming of social behaviors [12]. Causal findings demonstrating that social behaviors themselves can be directly impacted by epigenetic regulation are scarce. However, at a global level, exposure to L-methionine, a methyl donor that results in increased global DNA methylation, reduces aggressive behavior normally present in socially isolated male mice [15], suggesting that DNA methylation can be important for environmentally induced plasticity related to social function. In addition, mutant Tet1 mice with a deletion of exon 4 have lower expression of $\sim 180$ genes in the hippocampus, including specific isoforms of oxytocin receptor and hypermethylation of some CpG islands in the oxytocin receptor gene; this was associated with deficits in maternal care and increased aggression in the resident intruder task in females [16]. Human data from both healthy individuals and individuals with autism spectrum disorder also suggest that methylation of the oxytocin receptor gene correlates with social function $[17,18]$. The impact of manipulations to DNA methylation on the function of social circuits, whether global or specifically to social genes like the oxytocin receptor gene, have yet to be explored.

Our concept of DNA methylation as a highly stable epigenetic modification has shifted in recent years, predominantly as a result of research related to learning and memory, which has suggested that DNA methylation plays an important role in experience-dependent plasticity [19]. In addition, genome-wide analyses have shown that activity can rapidly affect the methylome in mature neurons [20]. These findings strongly suggest that DNA methylation may be a flexible tool by which the environment can impact the circuit remodeling and changes in synaptic connections required for dynamic social interactions; however, this has not yet been directly tested. 


\section{Conclusion \& future perspective}

There is substantial data suggesting that epigenetic regulation within social-related brain areas is critical for the function of these brain areas and subsequent expression of social behaviors including maternal behaviors, sexual behavior, pair bonding and aggression. However, there is little understanding as to how epigenetic alterations documented in these brain areas affect activity within these areas and plasticity within associated neural circuits. In addition, to this point, tools to specifically manipulate the epigenome in rodent brain are limited; however, there is some evidence that shows that manipulating epigenetic marks of specific genes can have significant effects on complex behavior [21]. With recent breakthroughs in molecular technologies [22], exciting new research avenues related to epigenetic manipulations and their impact on complex behavior are becoming increasingly available and will provide invaluable opportunities to probe the link between the epigenetic landscape, transcriptional regulation and circuit function.

\section{Financial \& competing interests disclosure}

RV Wheeler is supported by a National Sciences and Engineering Research Council of Canada Graduate Doctoral Scholarship (NSERC CGS-D) and the Franklin laboratory is supported by an NSERC Discovery Grant (RGPIN-2017-06490). The authors have no other relevant affiliations or financial involvement with any organization or entity with a financial interest in or financial conflict with the subject matter or materials discussed in the manuscript apart from those disclosed.

No writing assistance was utilized in the production of this manuscript.

\section{References}

1. Bludau A, Royer M, Meister G, Neumann ID, Menon R. Epigenetic regulation of the social brain. Trends Neurosci. 42(7), 471-484 (2019).

2. Chen P, Hong W. Neural circuit mechanisms of social behavior. Neuron 98(1), 16-30 (2018).

3. Meira T, Leroy F, Buss EW, Oliva A, Park J, Siegelbaum SA. A hippocampal circuit linking dorsal CA2 to ventral CA1 critical for social memory dynamics. Nat. Commun. 9(1), 4163 (2018).

4. Leroy F, Park J, Asok A et al. A circuit from hippocampal CA2 to lateral septum disinhibits social aggression. Nature 564(7735), 213-218 (2018).

5. Franklin TB, Silva BA, Perova Z et al. Prefrontal cortical control of a brainstem social behavior circuit. Nat. Neurosci. 20(2), 260-270 (2017).

6. Matsuda KI, Mori H, Nugent BM, Pfaff DW, Mccarthy MM, Kawata M. Histone deacetylation during brain development is essential for permanent masculinization of sexual behavior. Endocrinology 152(7), 2760-2767 (2011).

7. Bonthuis PJ, Patteson JK, Rissman EF. Acquisition of sexual receptivity: roles of chromatin acetylation, estrogen receptor-alpha and ovarian hormones. Endocrinology 152(8), 3172-3181 (2011).

8. Stolzenberg DS, Stevens JS, Rissman EF. Histone deacetylase inhibition induces long-lasting changes in maternal behavior and gene expression in female mice. Endocrinology 155(9), 3674-3683 (2014).

9. Wang H, Duclot F, Liu Y, Wang Z, Kabbaj M. Histone deacetylase inhibitors facilitate partner preference formation in female prairie voles. Nat. Neurosci. 16(7), 919-924 (2013).

10. Franklin TB, Russig H, Weiss IC et al. Epigenetic transmission of the impact of early stress across generations. Biol. Psychiatry 68(5), 408-415 (2010).

11. Karen C, Rajan KE. Social behaviour and epigenetic status in adolescent and adult rats: the contribution of early-life stressful social experience. Cell. Mol. Neurobiol. 39(3), 371-385 (2019).

12. Murgatroyd C, Patchev AV, Wu Y et al. Dynamic DNA methylation programs persistent adverse effects of early-life stress. Nat. Neurosci. 12(12), 1559-1566 (2009).

13. Turecki G, Meaney MJ. Effects of the social environment and stress on glucocorticoid receptor gene methylation: a systematic review. Biol. Psychiatry 79(2), 87-96 (2016).

14. Franklin TB, Linder N, Russig H, Thony B, Mansuy IM. Influence of early stress on social abilities and serotonergic functions across generations in mice. PLoS ONE 6(7), e21842 (2011).

15. Pinna G, Agis-Balboa RC, Doueiri MS, Guidotti A, Costa E. Brain neurosteroids in gender-related aggression induced by social isolation. Crit. Rev. Neurobiol. 16(1-2), 75-82 (2004).

16. Towers AJ, Tremblay MW, Chung L et al. Epigenetic dysregulation of Oxtr in Tet1-deficient mice has implications for neuropsychiatric disorders. JCI Insight 3(23), pii: 120592 (2018).

17. Gregory SG, Connelly JJ, Towers AJ et al. Genomic and epigenetic evidence for oxytocin receptor deficiency in autism. BMC Med. 7, 62 (2009). 


\section{Editorial Wheeler \& Franklin}

18. Jack A, Connelly JJ, Morris JP. DNA methylation of the oxytocin receptor gene predicts neural response to ambiguous social stimuli. Front. Hum. Neurosci. 6, 280 (2012).

19. Tognini P, Napoli D, Pizzorusso T. Dynamic DNA methylation in the brain: a new epigenetic mark for experience-dependent plasticity. Front. Cell Neurosci. 9, 331 (2015).

20. Guo JU, Ma DK, Mo H et al. Neuronal activity modifies the DNA methylation landscape in the adult brain. Nat. Neurosci. 14(10), 1345-1351 (2011).

21. Heller EA, Cates HM, Pena CJ et al. Locus-specific epigenetic remodeling controls addiction- and depression-related behaviors. Nat. Neurosci. 17(12), 1720-1727 (2014).

22. Liao HK, Hatanaka F, Araoka T et al. In vivo target gene activation via CRISPR/Cas9-mediated trans-epigenetic modulation. Cell 171(7), 1495-1507.e1415 (2017). 